\title{
Correction to: Satellite Images Combined with Field Data Reveal Negative Changes in the Distribution of Babassu Palms after Clearing off Amazonian Forests
}

\section{Mitja ${ }^{1}$ E. Delaître ${ }^{1}$ A. M. Santos ${ }^{1,2,3} \cdot$ I. Miranda $^{2} \cdot$ R. F. R. Coelho ${ }^{4}$ D. J. Macedo ${ }^{1}$ L. Demagistri ${ }^{1} \cdot$ M. Petit $^{5}$}

Published online: 9 January 2018

(c) Springer Science+Business Media, LLC, part of Springer Nature 2018

\section{Correction to: Environmental Management}

https://doi.org/10.1007/s00267-017-0965-6

The original version of this article unfortunately contained a mistake. The data in Table 4 were misaligned in the original version of this article. The correct version of Table 4 is given below.

The original article has been corrected.

The original article can be found online at https://doi.org/10.1007/ s00267-017-0965-6.

D. Mitja

danielle.mitja@ird.fr

1 UMR 228 ESPACE DEV (IRD-Institut de Recherche pour le Développement, UM-Université de Montpellier, UG-Université de Guyane, UR-Université de La Réunion, UA-Université des Antilles), MTD-IRD, 500 Rue Jean François Breton, 34093 Montpellier Cedex 5, France

2 ISARH-Instituto Ambiental e de Recursos Hídricos, UFRAUniversidade Federal Rural da Amazônia, CP.917, 66077530 Belém, Pará, Brazil

3 IEDAR-Instituto de Estudos em Desenvolvimento Agrário e Regional (UNIFESSPA-Universidade Federal do Sul e Sudeste do Pará), Folha 31 Quadra 07 Lote Especial, Nova Marabá, 68507-590 Marabá, Pará, Brazil

4 IFPA-Instituto Federal de Educação, Ciência e Tecnologia do Pará- Campus Castanhal, Br-316 km 62s/n. Bairro Saudades II -Cristo Redentor, 68740-970 Castanhal, Pará, Brazil

5 IRD, 911 avenue agropolis, 34394 Montpellier, France 
Table 4 Various factors impact on the babassu palm, in pasture

\begin{tabular}{|c|c|c|c|c|}
\hline & \multicolumn{2}{|l|}{ Fruits } & \multicolumn{2}{|l|}{ Life cycle } \\
\hline & Above the ground & Buried & $\begin{array}{l}\text { Seedlings and young } \\
\text { individuals stemless }\end{array}$ & $\begin{array}{l}\text { Young with stipe and } \\
\text { adults }\end{array}$ \\
\hline Sun & $\begin{array}{l}\text { Seed deterioration, } \\
\text { eliminates potential } \\
\text { individuals }\end{array}$ & $\begin{array}{l}\text { Very low deterioration } \\
\text { of seeds, little impact } \\
\text { on the individuals } \\
\text { number }\end{array}$ & Promotes growth & Promotes growth \\
\hline Fire & $\begin{array}{l}\text { Seed deterioration, } \\
\text { eliminates potential } \\
\text { individuals }\end{array}$ & $\begin{array}{l}\text { Very low deterioration } \\
\text { of seeds, little impact } \\
\text { on the individuals } \\
\text { number }\end{array}$ & $\begin{array}{l}\text { Slows the growth and } \\
\text { indirectly promotes } \\
\text { growth, no impact on } \\
\text { the individuals number }\end{array}$ & $\begin{array}{l}\text { Slow growth, can } \\
\text { eliminate some } \\
\text { individuals }\end{array}$ \\
\hline Hand weeding plot & - & - & $\begin{array}{l}\text { Slows the growth and } \\
\text { indirectly promotes } \\
\text { growth, no impact on } \\
\text { the individuals number }\end{array}$ & - \\
\hline $\begin{array}{l}\text { Specific chemical } \\
\text { weeding babassu }\end{array}$ & - & - & Eliminates individuals & - \\
\hline $\begin{array}{l}\text { Competition forage } \\
\text { grass }\end{array}$ & - & - & Slows growth & - \\
\hline Clearcutting & 一 & - & - & Eliminates individuals \\
\hline
\end{tabular}

